\title{
Effets de Présence: quels procédés pour le film-théâtre?
}

Sandrine Simeon

Franklin \& Marshall College - Lancaster, United States of America

RÉSUMÉ - Effets de Présence: quels procédés pour le film-théâtre? - Cet essai évalue les diverses méthodes qu'adoptent les créateurs de captations et de films réalisés à partir de spectacles scéniques afin de produire un effet de présence. La mise en scène, l'ontologie de l'image filmique, la pluralité d'interprétations des manifestations de présence, les connexions entre publics de la salle de théâtre et ceux de l'écran, font partie des éléments examinés. L'analyse conclut avec un plaidoyer pour le film-théâtre.

Mots-clés: Présence. Film-Théâtre. Mise en Scène. Public. Quatrième mur.

ABSTRACT - Effects of Presence: what methods for film-theater? - This essay assesses the diverse methods adopted by directors who film stage plays in order to produce an effect of presence. Mise-en-scène, the ontology of the filmic image, the many interpretations of presence occurrences, connections between the stage and the screen publics, are some of the elements examined. The analysis closes with a plea in favor of film-theater.

Keywords: Presence. Film-Theater. Mise-en-Scène. Public. $4^{\text {th }}$ Wall.

RESUMO - Efeitos de Presença: estratégias do filme-teatro? - O ensaio avalia os diferentes métodos de produção de um efeito de presença, adotados na gravação e na montagem de filmes realizados a partir de espetáculos cênicos. A encenação, a ontologia da imagem fílmica, a pluralidade de interpretaçóes das manifestaçóes de presença, as conexóes entre o público da sala de espetáculo e o do filme na tela, fazem parte dos elementos estudados. A análise termina com uma defesa do filmeteatro.

Palavras-chave: Presença. Filme-Teatro. Encenaçáo. Público. Quarta Parede. 
L’absence est absolue, mais la présence a ses degrés (Genette, 1972, p. 253).

Ionesco écrivit dans ses Notes et Contre-notes qu'il était insensible à la magie thêâtrale et ressentait un sentiment de malaise en tant que spectateur: "Ce qui me gênait au théâtre, c'était la présence sur le plateau des personnages en chair et en os. Leur présence matérielle détruisait la fiction" (Ionesco, 1975, p. 4). Peut-être Ionesco aurait-il mieux apprécié le spectacle s'il avait troqué son siège de salle de théâtre pour un siège d'où il aurait pu le regarder projeté sur un écran.

Or, contrairement à l'expérience d'Ionesco, c'est justement la présence corporelle des comédiens dans le hic et nunc de la représentation qui rend les arts dits "vivants" si uniques pour les praticiens de la scène et les théâtrophiles, et qui disparaîtrait lors de l'enregistrement audiovisuel. Néanmoins, comme l'annonce Gérard Genette, "L'absence est absolue, mais la présence a ses degrés" (Genette, 1972, p. 253). Elle peut en effet renvoyer à celle que partagent des comédiens et un public in situ et aux phénomènes de coprésence, à l'éventuelle aura d'une actrice, mais avec l'introduction des technologies au sein des arts vivants, elle peut tout aussi bien s'exprimer par le biais de médiatisations et doit donc être repensée. Cet essai considère les divers procédés qu'utilisent les réalisateurs de ces enregistrements, car si le film-théâtre ${ }^{1}$ interdit incontestablement tout phénomène de présence physique de par sa nature, il constitue toutefois un mode d'écriture en mesure de restituer à son public un effet de présence.

Ainsi que l'indique René Bourasa,

Dans les arts de la scène, le concept de présence est envisagé non seulement en fonction du sujet, mais également en fonction de l'acteur. Il est construit autour de sa corporalité: l'intensité de sa présence physique est au cœur de l'acte performatif. Elle renvoie à sa force attractive ou à son charisme capable de capturer l'attention du public (Bourassa, 2013, p. 132).

En conséquence, pour certains, la médiatisation entraîne inéluctablement l'altération de cette présence essentielle. Walter Benjamin avait déjà noté, dans L'CEuvre d'art à l'époque de sa reproductibilité technique, que la reproduction technique dépréciait "l'aura" d'une ouvre car, décontextualisée, elle perdait alors sa valeur unique, originelle et première: "[...] le hic et nunc de l'original constitue ce qu'on appelle son authenticité. [...] En multipliant les exemplaires, elle substitue à son occurrence unique son existence 
en série" (Benjamin, 2000, p.13; p. 15). L'unicité de l'œuvre est ainsi détruite par sa reproduction, une multiplication responsable de sa démystification, et par extension nous pourrions dire qu'elle perd de sa présence.

De même, Pascal Bouchez envisage la présence avant tout comme le produit d'un processus de contextualisation qui engendre un échange réflexif entre émetteur et récepteur: "Le théâtre, comme processus autopoiétique, n'existe pas pour être 'vu' (quelle extraordinaire réduction!), mais pour être expérimenté, vécu, partagé”. Puisque sa théorie de l'autopoïèse théâtrale implique, entre autre, que la communication, la production du sens, émerge de l'interaction en constante évolution entre un sujet et son environnement, Bouchez rapporte que "nous sommes tous des êtres résonateurs avant d'être des êtres raisonneurs". L'individu peut donc transmettre des “[...] signaux qui peuvent modifier non seulement l'humeur et les émotions, mais aussi les niveaux hormonaux, la fonction cardiovasculaire". Bouchez conclut que d'innombrables études empiriques ont mis en lumière ce processus de réflexivité des émotions chaque fois que des individus sont en présence les uns des autres et que "[...] l'apport du récepteur à la construction du sens sera plus important dès lors qu'il sera en résonance [...] avec l'émetteur" (Bouchez, 2007, p. 39-40). Ainsi, lors du filmage d'un spectacle, dû au facteur de médiation engendré par la caméra, la transposition du texte spectaculaire sur support audiovisuel suppose un écart entre les deux entités essentielles de l'autopoïèse théâtrale, les comédiens et le public de la salle. Selon cette hypothèse, l'instance réceptrice du film-théâtre ne peut vivre physiquement ses rapports avec la scène. En effet, la résonance suppose une synchronie, un même espace-temps, qui disparaît forcément puisque le filmthéâtre est un événement vécu lors de son visionnement, qui n'est pas le moment de la présentation du texte spectaculaire. A fortiori, l'échange de nature biologique entre l'émetteur et le récepteur - tous deux simultanément acteurs et spectateurs - est altéré. La sémiose théâtrale est alors appauvrie, voire inexistante. Malgré les velléités de réalisme du médium filmique, le film-théâtre n'est plus qu'une empreinte qui s'adresserait à la raison, et n'est plus en résonance avec le public. La présence, ainsi définie par Bouchez, n'est concevable que pour une audience en mesure de ressentir le texte spectaculaire de visu.

Pourtant, Bourassa souligne que 
Nous sommes présents là où notre attention se porte, que ce soit dans un environnement physique réel ou par le biais d'un dispositif médiatisé. [...] Dans les arts de fiction, l'immersion vécue au sein d'un monde imaginaire constitue un puissant attracteur de l'attention du spectateur, qui engage son activité mentale. Lorsque l'action dramaturgique d'une performance ou d'un film le captive, son attention est absorbée entièrement par le devenir fictif des personnages ou des situations qui le rendent présent à l'acte de spectature (Bourassa, 2013, p. 130).

Ainsi, il devient légitime d'affirmer que la présence d'une actrice, son charisme, transcendent les limites spatio-temporelles de l'écran. Bien que le spectateur soit physiquement séparé des comédiens et qu'il sache que le moment de la présentation du texte scénique est effectivement révolu, il vit l'événement psychiquement au présent. La charge émotionnelle l'atteint comme s'il faisait l'expérience de cette émotion dans un espace-temps concordant avec celui du public qu'il entend et voit applaudir. Il ressent ce qui est montré par la médiatisation des images comme si celle-ci était inexistante; il répond au spectacle comme si l'écran ne créait pas de distance spatiotemporelle entre le présent de la représentation et le présent de sa réception.

Du point de vue de la réception, le dispositif scénique peut influencer l'expérience des publics de l'écran et celle-ci peut être modifiée selon que son alter ego est visible ou non. Néanmoins, le choix d'inclure ou d'évincer le public de l'écran dépend parfois de contraintes matérielles. Pour la captation de la mise en scène de Frankenstein de Danny Boyle par Tim Van Someren (2010), l'espace scénique circulaire de l'Olivier Theater au National Theater de Londres autorisait la disposition de l'audience en arc de cercle, ce qui prévenait en contrepartie les prises de vue purement frontales de la scène et incluait inévitablement l'audience dans le champ de la caméra. En outre, la présence d'un luminaire immense situé au-dessus de la scène obligeait la caméra à rester en retrait. En revanche, le théâtre à l'Italienne ${ }^{2}$ d'où Martin Fraudreau filme la mise en scène du Bourgeois gentilhomme de Benjamin Lazar en 2004 lui aurait permis d'occulter complètement le public. Or, celui-ci est présent à plusieurs reprises à l'intérieur des limites de l'écran - que Fraudreau l'ait inclus à dessein ou parce que, pour montrer la rampe bordée de chandelles, il a forcément dû reculer sa caméra et n'a alors pu éviter de garder dans son champ les sièges des premiers rangs. Enfin, pour la version filmique d'Hamlet réalisée en 2010 par Robin Lough et d'après une mise en scène de Nicolas Hytner, l'audience n'est visible et audible - des ri- 
res parcimonieux à peine discernables - que lorsque le spectacle débute, et durant l'entracte. Bien que ce choix ambitionne peut-être une appropriation plus immédiate de la scène par le spectateur de l'écran, ce procédé peut engendrer une décontextualisation par rapport à l'événement dans son ensemble. Dans ce cas, les spectateurs qui m'entouraient dans la salle de cinéma lors de la retransmission de Hamlet par le National Theater Live ${ }^{3}$ me semblaient bien plus présents que ceux de la salle du National Theater, et ceux-ci ont applaudi à la fin de la retransmission, comme ils l'auraient fait, eussent-ils assisté en chair et en os à l'interprétation scénique de Nicholas Hytner. La présence des comédiens communique donc une émotion qu'il est possible de vivre au présent, même si ce moment se distingue géographiquement et temporellement de celui vécu par le public du spectacle ${ }^{4}$.

Nous pourrions alors poser comme axiomatique que la présence se manifeste de manière multiple et adopter la définition de "l'effet de présence" avancée par Josette Féral afin de concrétiser les diverses manifestations de la présence, notamment dans le cas de dispositifs médiatisés: "L'effet de présence est ce sentiment qu'a un spectateur que les corps ou les objets offerts à son regard (ou à son oreille) sont bien là, dans le même espace et le même temps que ceux dans lequel il se trouve alors qu'il sait pertinemment qu'ils sont absents" (Féral; Perrot, 2012, p. 26). Bourassa a ensuite parfait cette détermination en ajoutant que les effets de présence décrivent

[...] les procédés en cause dans l'induction du sentiment de présence chez le sujet en situation d'expérience médiatisée. [...] Le terme 'effet' désigne ce qui agit, ce qui opère. Il désigne également ce qui met en valeur un phénomène par ostension. Du côté du dispositif matériel, il renvoie à des phénomènes d'accentuation ou d'altération de la présence par des procédés d'augmentation, d'amplification, de grossissement, de simulation, de substitution, de subterfuge ou de déplacements (Bourassa, 2013, p. 133).

Ces procédés d'augmentation et d'amplification évoquent certainement les réflexions de Gérard-Denis Farcy et René Prédal qui postulent qu'il y aurait "[...] une distance au-delà de laquelle la présence du comédien se diluerait" (Farcy; Prédal, 2001, p. 18), corroborant ainsi le refus d'André Bazin d'opposer texte scénique et film-théâtre sur la seule notion de présence. Dans Théâtre et cinéma Bazin suggère en effet que la présence est récupérée grâce à la proximité artificielle que permettent les plans rapprochés, et la multiplication des points de vue (Bazin, 1951, p. 234). Il souligne alors 
un nouveau paradoxe de l'acteur de film-théâtre: alors qu'il est techniquement inaccessible, absent de l'espace de la salle d'où est visionné le film, l'acteur semble néanmoins plus présent grâce à la proximité des gros plans qui suscitent une intimité physique avec lui. Par conséquent, si l'on admet que la présence de l'acteur sur scène assure une certaine immédiateté, le même acteur perçu d'une position éloignée dans la salle a tendance à perdre de sa matérialité - bien que cet argument puisse être également nuancé en fonction de la topographie du théâtre et de la distance entre le siège du spectateur et la scène.

Ces effets de grossissement sont fréquemment utilisés dans les filmsthéâtre: Martin Fraudreau, pour le filmage du Bourgeois gentilhomme en 2004, exploite les plans rapprochés afin d'amoindrir la distance entre la scène et le public de l'écran. Le réalisateur tire également profit d'effets de simulation permettant de susciter une participation active de la part de l'audience filmique. Ainsi, il fait jouer au montage un rôle fondamental afin d'exprimer l'immédiateté inhérente au texte scénique. Par exemple, dans le texte de Molière, le maitre de musique invite ses musiciens - ainsi que son auditoire - par cette réplique illocutoire: "Venez, entrez dans cette Salle, et vous reposez là, en attendant qu'il vienne" (Molière, 2010, p. 265). Sur la scène, cette invitation est encore plus prégnante par la manière dont ce personnage apparaît, littéralement. En effet, celui-ci - puis le maître de danse ensuite - entre par un accès disposé face au public à l'extrémité de la scène, mais qui passe jusqu'alors inaperçu car intégré au décor. Les maittres de musique et de danse, par leur entrée inattendue, surprennent donc le spectateur de la salle qui les voit émerger par ces ouvertures. A ce moment précis de l'action, Fraudreau choisit de montrer les entrées de ces personnages par un changement de plan. Fraudreau crée ainsi l'immédiateté de la surprise à l'aide d'un montage idoine: le premier plan rapproché décrit le geste du maître de musique ouvrant la porte; le cadrage de la caméra double l'encadrement de cette ouverture (Figure 1). 


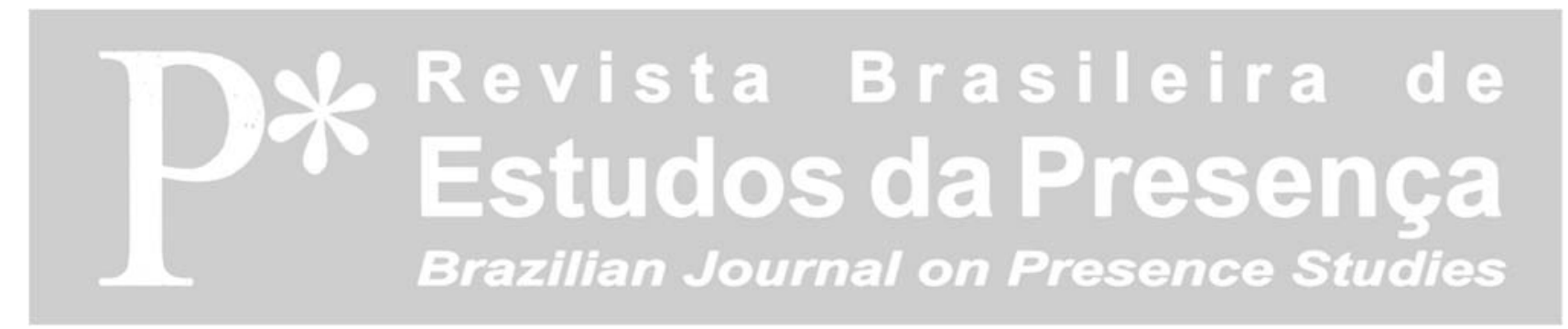

E-ISSN 2237-2660

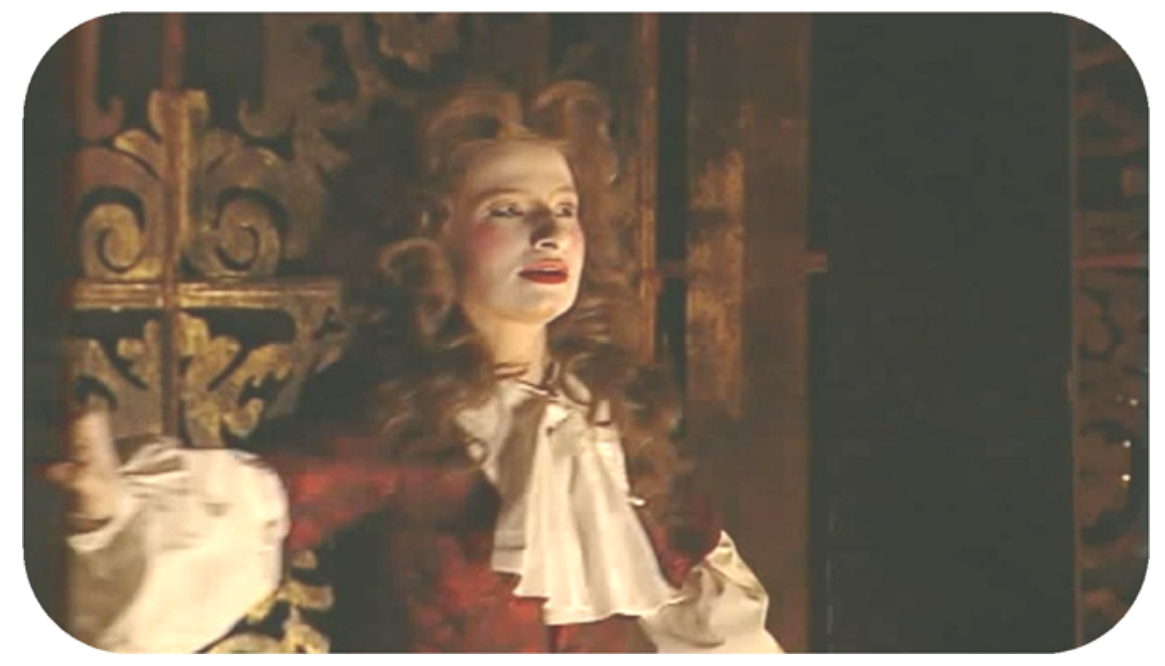

Figure 1 - Capture film, Le Bourgeois gentilhomme, Martin Fraudreau (2004).

Suit alors un plan d'ensemble de la scène permettant ainsi au spectateur du film-théâtre de s'orienter et de voir les deux personnages surgis des décors dans leur contexte scénique (Figure 2).

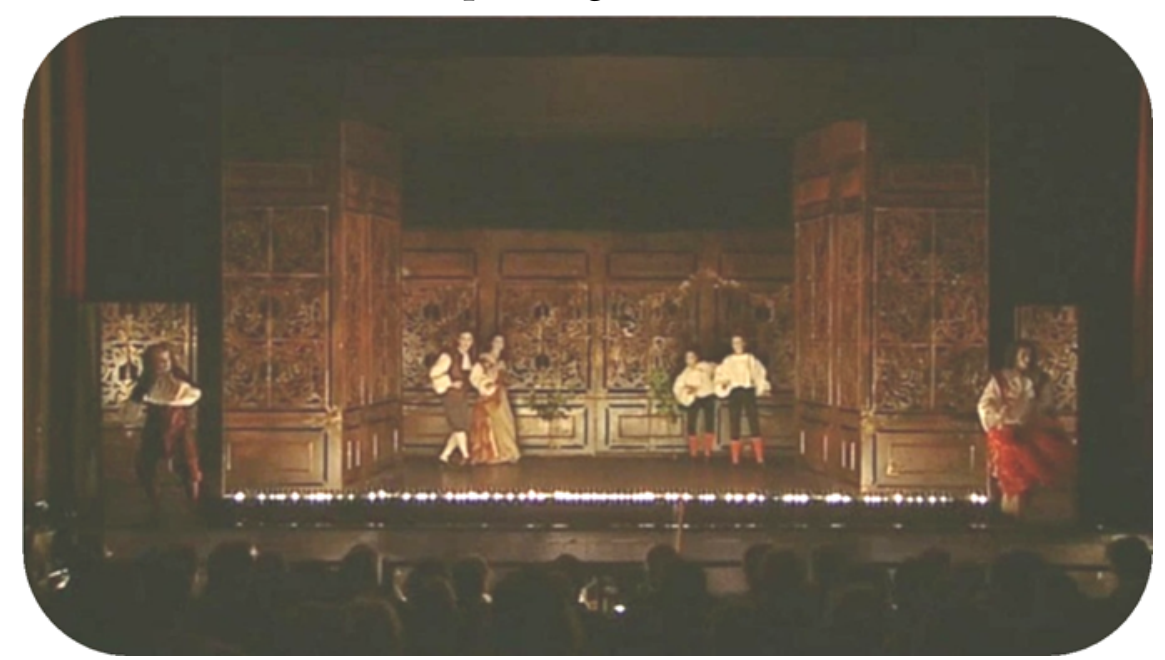

Figure 2 - Capture film, Le Bourgeois gentilhomme, Martin Fraudreau (2004).

La manière dont les entrées en scène des maitres de danse et de musique sont mises en scène témoigne d'un choix représentatif de filmage de la part du réalisateur qui contribue à l'effet d'immédiateté destiné spécifiquement au spectateur du film. Fraudreau crée un effet de surprise destiné au public de l'écran similaire à celui de la salle à l'aide de la rhétorique filmique - en l'occurrence: échelle de plan, cadrage et montage. Ces choix de filmage sont toujours justifiés par l'écriture du film-théâtre sans néanmoins enfreindre les spécificités du texte scénique. En effet, d'une part la réalisation de Fraudreau privilégie le point de vue de son instance spectatrice puisqu'il lui offre une proximité qui supplée à son absence à l'aide du plan rapproché 
- ce que suggérait Bazin. De l'autre, si le réalisateur favorise une énonciation avant tout filmique, sa réalisation témoigne d'un refus de morcèlement arbitraire de l'espace scénique, et privilégie souvent la profondeur de champ, l'ensemble plutôt que la partie - comme en témoigne le plan d'ensemble suivant immédiatement le plan rapproché du maître de musique. Fraudreau relaie ainsi les spécificités du texte scénique sur le terrain du médium filmique, concourant ainsi à l'effet de surprise d'abord mis en scène par Benjamin Lazar.

Les exemples suivants mettent en lumière les effets de substitution et de subterfuge qu'expose Bourassa. Pour La Cantatrice chanve, Phèdre, et 1789, l'audience de la salle est mise à contribution de manière à convoquer l'attention de son homologue de l'écran dans l'objectif de lui communiquer l'autopoïèse constitutive de l'événement scénique.

Dans la mise en scène de La Cantatrice chauve d'Ionesco par Jean-Luc Lagarce, le rôle métaréflexif conféré au personnage de Mary sert de relais entre les deux publics de la salle et de l'écran, notamment lorsqu'elle déclame directement à l'auditoire les notes de bas de page - rajoutées par Ionesco dans les éditions ultérieures du texte dramatique où il commente la toute première mise en scène de son texte ${ }^{5}$. Pour ces apartés au public, le réalisateur du film du même nom, Vincent Bataillon, a systématiquement choisi le regard caméra afin de placer son spectateur dans la position de celui de la salle et d'engager son attention. Par ailleurs, Lagarce avait révélé à ses spectateurs les divers dénouements auxquels avaient pensés Ionesco: Madame Smith prend la parole et s'adresse directement à la salle pour annoncer que la bonne devait faire une dernière apparition et prévenir que: le dîner était prêt; des gendarmes devaient fusiller des spectateurs et ordonner au public de quitter la salle; l'auteur lui-même viendrait insulter l'audience. Ionesco, n'ayant pas trouvé la fin idéale, suggère que la pièce recommence. Lagarce prolonge alors l'idée de Ionesco et met encore une fois la salle à contribution lorsque ses comédiens suggèrent un dénouement supplémentaire: silencieux, ils restent assis face à la salle. Dans le film, la caméra opère alors un zoom arrière afin d'exposer une audience perplexe, qui finit par applaudir. Mais les comédiens se lèvent et poussent des spectatrices sur la scène. Or, bien que le public n'ait pas, jusqu'à ce moment-là, été inclus dans le cadre de l'objectif, Bataillon choisit de l'impliquer lorsque les acteurs pénètrent physiquement l'espace de la salle. Dès ce moment, en effet, 
les prises de vue alternent entre la salle et la scène. Les procédés de filmage et de réalisation sont ainsi adaptés dans l'objectif de communiquer au récepteur filmique l'expérience de son homologue de la salle: l'incertitude de ce dernier est exprimé à l'aide d'un zoom arrière et une série de gros plans transmet les réactions individuelles.

Une telle rupture du quatrième mur, aussi inattendue, est également simulée dans la mise en scène de Phèdre de Patrice Chéreau. Stéphane Metge réalise le film en 2004 à partir de plusieurs présentations en public au Théâtre de l'Odéon. La configuration de ce théâtre ne semble pas si différente de celle des Bouffes du Nord où Ricardo Aronovitch avait tourné Hamlet sans public en 2002 dans une mise en scène de Peter Brook. C'est donc un parti pris de la part de Metge de tourner le film en public. Contrairement au Hamlet d'Aronovitch, les plans rapprochés font exception dans le film de Metge, et les plans moyens dominent quand la caméra s'avance vers les comédiens - à l'exception faite du personnage de Phèdre et du cadavre d'Hyppolite lorsque celui-ci est exhibé sur scène. Cette mise à distance est dictée par au moins deux raisons: la topographie de l'espace scénique et la volonté d'inclure le public durant le tournage (Figure 3).

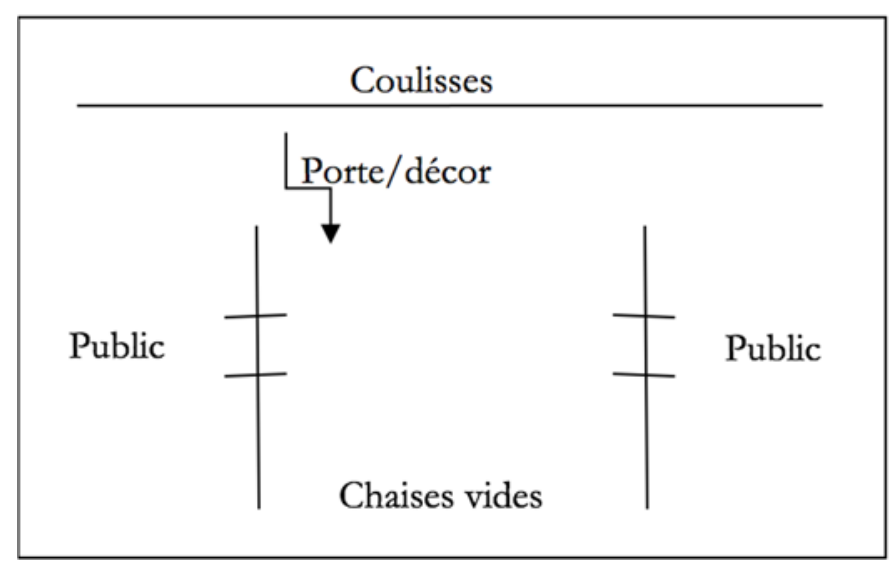

Figure 3 - Topographie de la scène.

Le public enserre la scène sur deux bords et se fait face, les décors occupent les deux autres - les coulisses d'un côté et quelques chaises vides ${ }^{6}$ de l'autre -, contraignant la caméra à se retrancher vers une ouverture donnant sur les coulisses ou dans les gradins. Bien que les moyens optiques des caméras autorisent la proximité, les gros plans refouleraient le public en dehors des limites du cadre de l'objectif et nieraient sa présence. Or l'espace de la salle fait partie intégrante du texte spectaculaire de Chéreau, et le pu- 
blic, sans pourtant y participer de manière proprement interactive, y joue néanmoins un rôle crucial.

Les premières images exposent le public alors qu'il s'installe dans les gradins; elles sont prises à partir d'un accès aux coulisses, la caméra est fixe. Un tel exorde anticipe le rôle de l'audience dont la présence est non seulement perceptible sur l'écran, mais concrétise également un lien et un prolongement ostensible avec l'espace scénique. Ainsi Hippolyte arrive-t-il par les gradins sans signes annonciateurs - les trois coups de brigadier un peu démodés, le lever du rideau quand il y a en a un, une diminution de l'éclairage. La mise en scène d'une telle apparition double l'in media res du texte dramatique de Racine: "Le dessein en est pris, je pars, cher Théramène, / Et quitte le séjour de l'aimable Trézène" (Racine, 2010, p. 821). Plus tard, Théramène s'assied parmi le public, et Thésée va quasiment pleurer sur les genoux d'une spectatrice. L'audience du premier rang ne s'attend pas à une si brutale pénétration de son domaine; elle s'écarte par réflexe. Plus tard encore, un spectateur, dès lors averti, réagit vite: il retire son programme de la marche sur laquelle Hyppolite vient se jeter. La mise en scène de Chéreau impose donc une présence incontestablement charnelle des acteurs et postule ainsi une coprésence fortement marquée, puisque les personnages évoluent dans un espace qui comprend la salle - ou ce qui est symboliquement délimité comme domaine appartenant à celle-ci.

L'interférence occasionnée par la proximité physique des comédiens produit au moins deux effets dramaturgiques. D’une part, ce procédé interactif entre les comédiens et le public remplit les trois fonctions, élaborées par Vuillemin: de focalisation, didactique et d'effet de réel. Ainsi, en investissant l'espace de la salle, les acteurs attirent l'attention sur leur présence corporelle - fonction de focalisation. Le spectateur prend alors conscience de sa propre position au sein du processus créatif: “[...] l'acte réceptif intègre le processus de la production qui ne saurait [...] se concevoir sans la participation d'une instance réceptrice" (Vuillemin, 2010, p. 124) - fonction didactique. Puis, parce qu'il saute lui-même la rampe, le personnage impose une proximité avec son public qui estompe en même temps le partage entre réalité et fiction - fonction d'effet de réel. D'autre part, cette interférence force l'intégration du système scène/salle, et permet d'unifier les deux composantes inter-réceptives essentielles à l'autopoï̀se théâtrale. Toutefois, ce dispositif revendique une interaction avec le public sans que les comédiens 
s'adressent à lui directement. A l'instar du théâtre dramatique de Racine qui interdit l'adresse au public afin de maintenir l'illusion fictionnelle, l'interprétation de Chéreau ne propose pas une franche rupture du quatrième mur. Même si la fiction fait brusquement irruption dans l'espace de réalité du public, la séparation entre l'univers imaginaire et le monde réel reste intacte. En effet, à la différence du théâtre épique où le quatrième mur est rompu à dessein, afin de briser l'illusion de réalité dans laquelle s'oublierait l'instance spectatrice, les comédiens de Phèdre ne prennent jamais à partie la salle, ni ne regardent dans l'objectif de la caméra. Si la proximité entre eux et certains spectateurs relève parfois de l'intimité ${ }^{7}$, aucun regard n'est échangé, ni aucune parole. Le mode d'interaction adopté par Chéreau n'engendre par conséquent aucune interruption de la diégèse.

Ainsi la mise en scène de Phèdre favorise-t-elle une attitude de "dénégation" que Pavis définit comme un phénomène dialectique au sein duquel le spectateur est en position de perpétuel va et vient entre identification et distanciation, entre illusion et réalité: "[...] il y a à la fois séparation entre scène et salle et chassés-croisés constant entre eux" (Pavis, 1996, p. 277). Dans le texte spectaculaire de Chéreau, les comédiens misent sur cette dénégation sans que ne soit théoriquement jamais rompu le quatrième mur, comme s'ils étiraient cette membrane invisible dans plusieurs directions sans que jamais celle-ci ne perce. Les spectateurs, en retour, s'efforcent de conserver leur pacte d'adhésion.

Les procédés de focalisation exploités par Chéreau sont relayés à l'aide des techniques de tournage de Metge, indéniablement conçues pour amplifier la perspective et la participation de l'audience écranique. En outre, non seulement Metge privilégie le point de vue du public de la salle en multipliant les perspectives à partir des deux parties latérales où se trouvent les gradins, mais il offre également un regard inédit au spectateur de l'écran - par une ouverture vers les coulisses - et qui reste par conséquent interdit à celui de la salle. Ce regard est pourtant motivé puisqu'il est celui du metteur en scène, comme on s'en aperçoit de manière rétrospective, alors que la caméra se focalise sur Chéreau qui sourit dans l'ombre à l'entrée des coulisses, juste avant le générique de fin. L'ajout du point de vue du metteur en scène porte en soi un surcroît de présence et une marque supplémentaire de réflexivité: Chéreau lui-même était là et son regard, par voie de relais, enrichit celui du public de l'écran. 
La mise en scène de Chéreau et la réalisation de Metge incarnent par conséquent, chacune à leur manière, la présence physique et psychologique des acteurs en coprésence avec leur audience. Le filmage de Phèdre satisfait ainsi aux besoins de l'autopoï̀se théâtrale puisque le public de la salle est non seulement perceptible - visible et audible par l'audience filmique -, mais il est surtout mis à contribution. Chéreau intensifie effectivement la relation des deux publics de la salle et de l'écran, lorsqu'il rend la présence du premier d'autant plus sensible que le second s'y projette inévitablement et réagit de manière virtuelle aux moments où les comédiens viennent envahir l'espace spectatoriel de la salle. Le public scénique sert alors d'embrayeur pour celui de l'écran, et permet de placer l'événement scénique dans une réalité spatio-temporelle afin de concrétiser la relation scène/salle.

Ce processus de substitution est encore exploité pour le tournage de 1789 par Ariane Mnouchkine en 1973. Mnouchkine laisse sa caméra participer à l'expérience des spectateurs présents à son spectacle pour offrir à l'audience de l'écran les perspectives variées de leurs homologues de la salle: le technicien circule entre les plateaux scéniques, la caméra à l'épaule. Ce procédé génère une image instable qui, parce qu'elle semble épouser les mouvements des participants, suggère ainsi une certaine subjectivité. Cet effet d'immersion dans l'action permet de renforcer l'identification du public de l'écran à celui de la salle. En outre, les faux raccords de montage, et le fait que l'objectif soit obstrué à plusieurs occasions, transmet une impression de grande mobilité et, parfois même, de confusion qui communiquent encore l'immédiateté du texte spectaculaire (Figure 4).

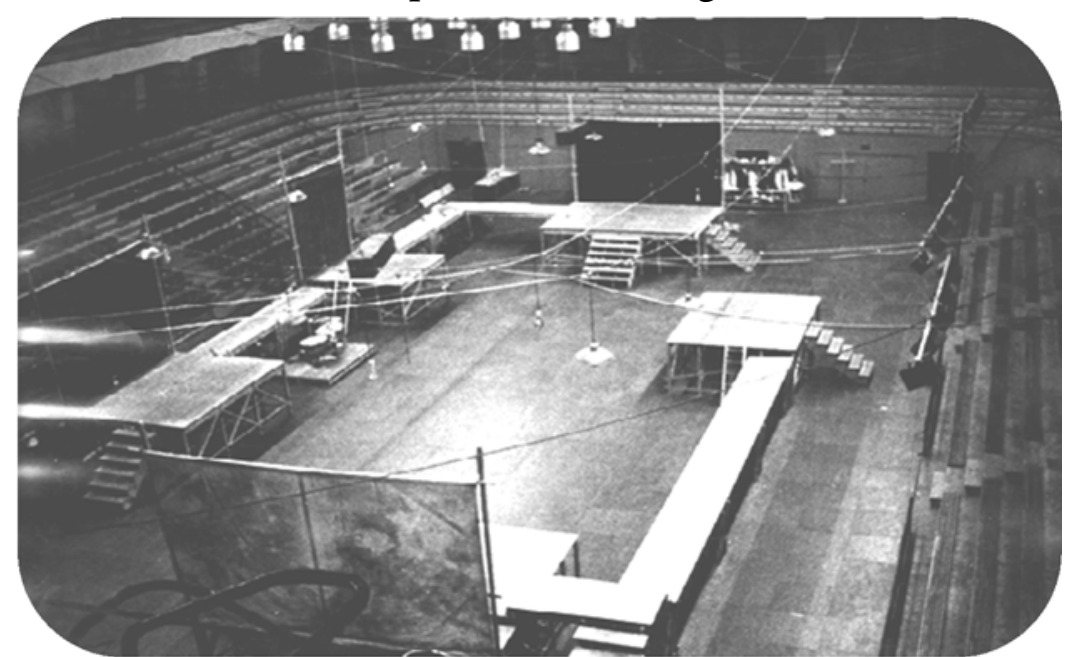

Figure 4 - Photo. Dispositif scénique de 1789. Source: Théâtre du soleil ${ }^{8}$. 
Mnouchkine a depuis exploré diverses méthodes d'enregistrement, les adaptant à chaque fois selon le dispositif scénique de ses mises en scène. Pour le Dernier Caravansérail, elle repense drastiquement l'enregistrement et exclut le public du tournage afin d'intensifier l'identification de celui de l'écran. Elle met ainsi à profit deux concepts théorisés par Bazin: celui de proximité déjà mentionné, et celui de l'ontologie de l'image photographique $^{9}$ qui contribue à l'effet de subterfuge, autre élément participant aux effets de présence à l'œuvre dans ce film.

La première partie du spectacle est jouée lors du festival d'Avignon en 2003. Le film qu'elle réalise est tourné sans public à la Cartoucherie en 2005, puis est projeté dans sa totalité le 10 juillet 2006 dans la Cour d'Honneur, en Avignon. À l'instar du texte spectaculaire, le film débute avec la traversée d'un fleuve, à cette différence près que la première image est un plan rapproché sur le visage d'un des personnages, un passeur. Ainsi, dès la première scène, les points de vue du public de la salle et celui de l'écran reçoivent un traitement totalement différent. L'audience de la Cartoucherie assiste à la traversée du fleuve par des personnages qu'elle contemple de manière latérale et à une certaine distance, variant selon sa position dans le théâtre (Figure 5):

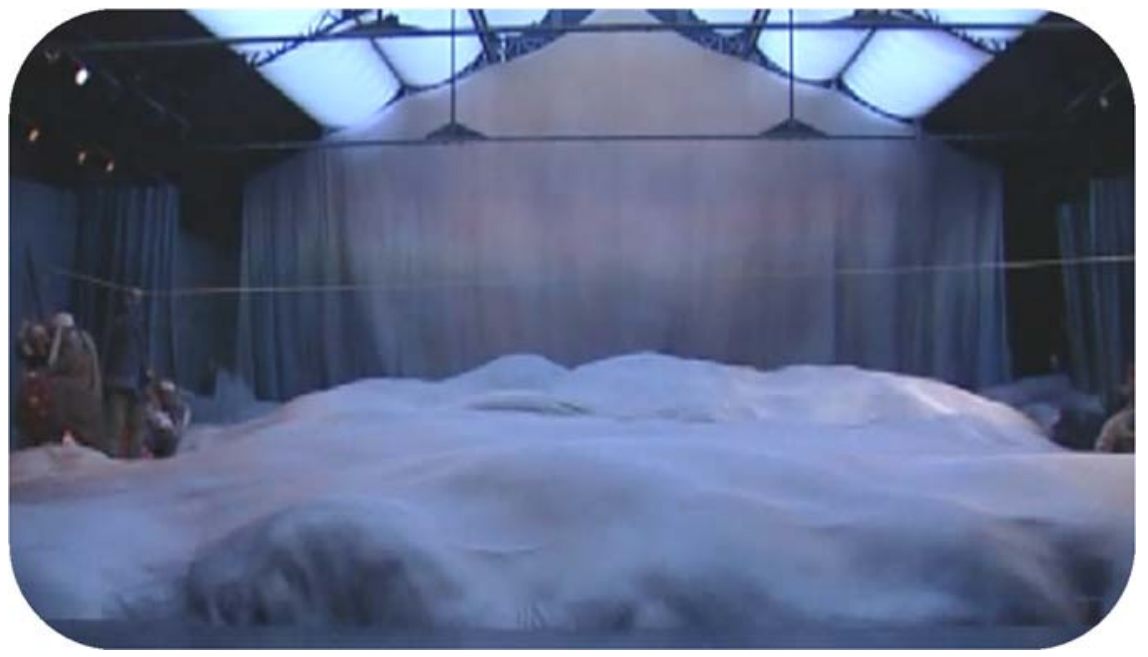

Figure 5 - Capture DVD. Complément de programme: Et au théâtre, c'était comment? Le Dernier Caravansérail, Ariane Mnouchkine (2006).

Celui de l'écran se trouve face à face avec eux (Figure 6): 


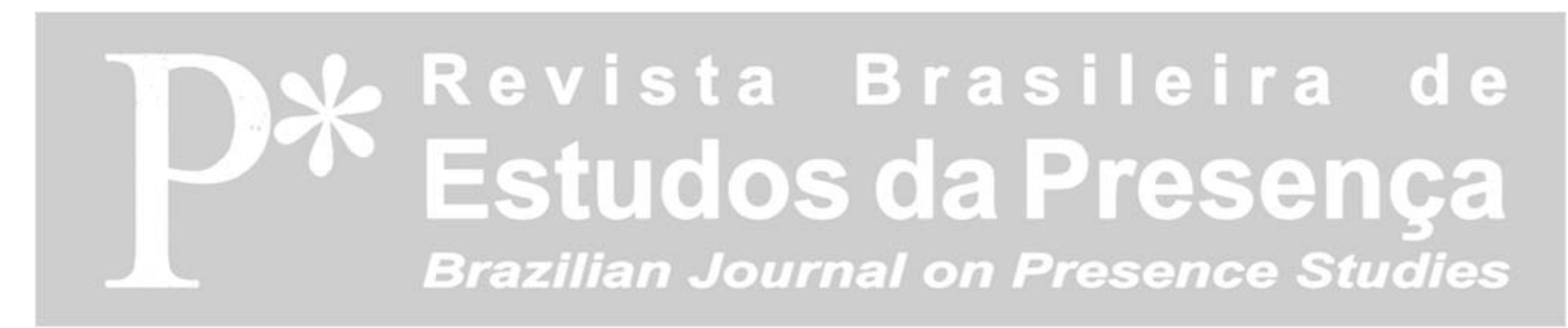

E-ISSN 2237-2660

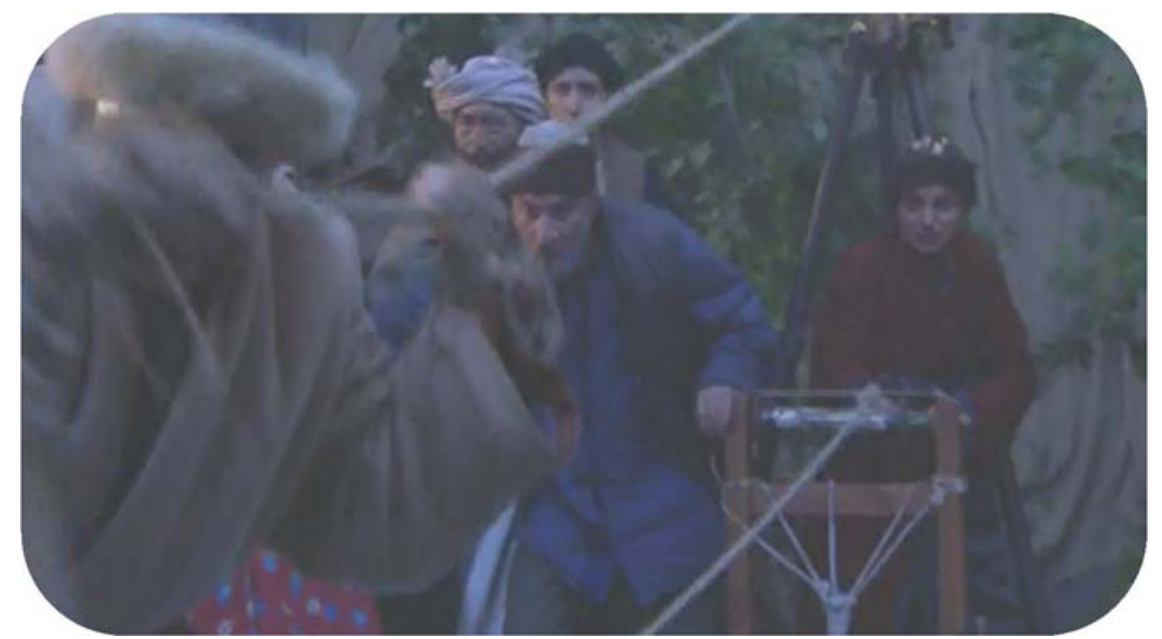

Figure 6 - Capture film, Le Dernier Caravansérail, Ariane Mnouchkine (2006).

Ce changement de perspective est crucial pour deux raisons. D'abord, puisque le spectateur de l'écran est physiquement absent de la salle et qu'il n'a pas accès à la présence matérielle de l'acteur, il doit, de manière à mieux pouvoir participer à l'action, se rapprocher virtuellement des comédiens. La présence est alors récupérée de deux manières: grâce à la proximité artificielle que procure la caméra et par la multiplication du point de vue. Ensuite, parce que l'audience écranique est positionnée dans l'axe du fleuve, les personnages entrent et sortent du cadre de l'objectif comme ceux de L'Arrivée d'un train en gare de la Ciotat $^{10}$, à savoir qu'ils décrivent des mouvements de l'arrière-plan vers le premier plan - l'opérateur des frères Lumière fait également face aux rails avec un point de fuite vers le fond de l'écran (Figure 7).

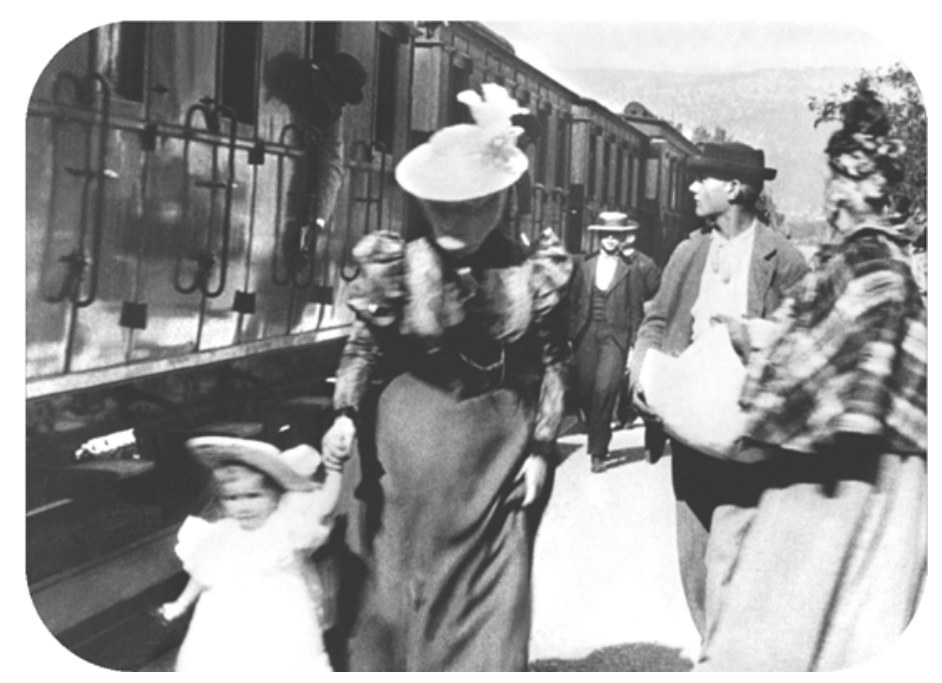

Figure 7 - Capture Film Lumière №653: L'Arrivée d'un train en gare de la Ciotat (France) (C) Institut Lumière. Frères Lumière $(1895)^{11}$. 
Ce choix de composition a deux incidences sur l'implication de l'instance réceptrice dans l'action. D'une part, la diversité des échelles de plan assure une composition plastique qui met en volume la bidimensionnalité de l'image et renforce l'illusion de sa tridimensionnalité grâce à la profondeur de champ créée par la perspective afin de produire un espace imaginaire conforme à la perception optique du monde réel (Figure 8).

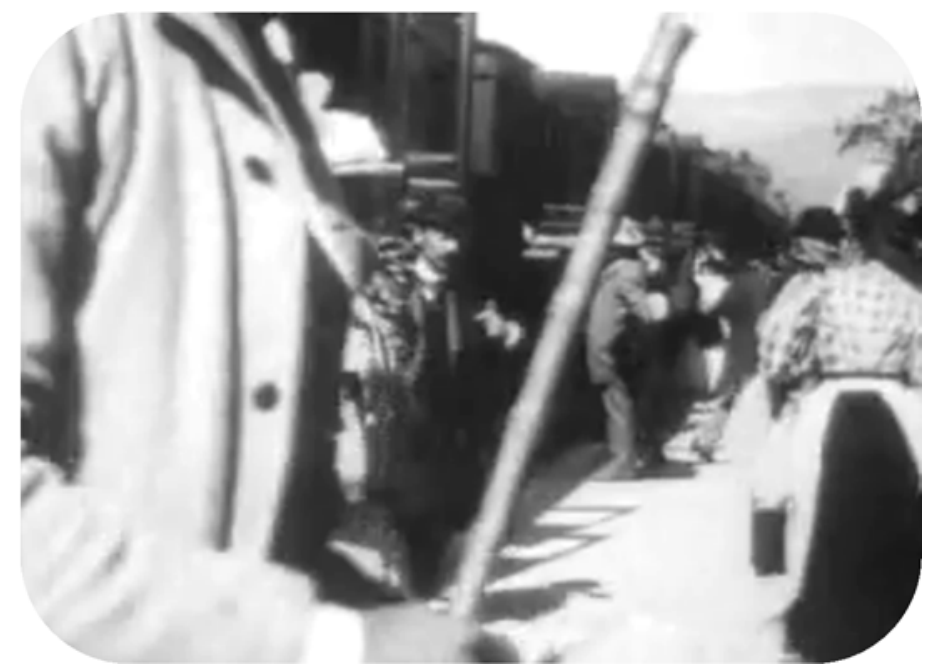

Figure 8 - Capture Film Lumière $\mathrm{N}^{\circ} 653$ : L'Arrivée d’un train en gare de la Ciotat (France) $\odot$ Institut Lumière. Frères Lumière $(1895)^{12}$.

D'autre part, l'effet de grossissement créé par le corps du personnage en premier plan qui déborde largement les limites du cadre, convoque l'attention du spectateur puisque l'espace du hors-champ, invisible, est alors concrétisé. Selon Bazin, au cinéma, le monde est rendu comme un reflet dans un miroir, c'est-à-dire que les limites de l'écran se calquent sur celles de ce miroir. Le hors-champ s'ouvre à l'infini, laissant croire à un monde réel aussi ouvert que celui du spectateur. Ce dernier est happé par l'espace infini de ce monde, l'écran agissant comme une "fenêtre sur le monde" (Bazin, 1951, p. 244). Le cadre acquiert ainsi une fonction centrifuge et ce débordement crée un lien entre le monde imaginaire visible à l'intérieur du cadre avec celui, réel et hors-cadre, du spectateur - ce choix de composition permet ainsi d'outrepasser les contraintes du quatrième mur. En effet, l'espace compris entre les limites de l'écran n'est que relativement et virtuellement clos et, ainsi que l'affirme Gilles Deleuze, il est communiquant: "Tel est le premier sens de ce qu'on appelle hors-champ: un ensemble étant cadré, donc vu, il y a toujours un plus grand ensemble, ou un autre avec lequel le premier en forme un plus grand, et qui peut être vu à son tour, à condition de susciter un nouveau hors-champ" (Deleuze, 1983, p. 29). Par 
conséquent, si tout cadrage souligne l'existence d'un hors-champ, celui-là ne discrimine pourtant pas nécessairement entre l'espace imaginaire du personnage et celui, matériel, du spectateur. Le spectateur sait qu'il n'appartient pas au monde imaginaire, mais la potentialité d'un espace ouvert à l'infini réduit paradoxalement l'écart entre l'espace réel et l'espace fictionnel et invite le spectateur à se projeter mentalement dans l'univers filmique: "Dans un contexte médiatique, l'attention oscille entre la matérialité du monde physique où se situe le corps du sujet, la médiatisation au moyen de dispositifs technologiques et la dimension symbolique dans l'espace imaginaire" (Bourassa, 2013, p. 130). Cette invitation à vaincre la séparation entre monde fictionnel et monde réel s'effectue encore selon un procédé authentifié par Batista Alberti qui accorde à des personnage-témoins un rôle de relais entre une scène et son spectateur ou, en l'occurrence, entre le champ et le hors-champ. Le personnage dont le corps obstrue la moitié de l'écran est ce personnage-embrayeur ${ }^{13}$ qui inclut le spectateur de l'écran au monde imaginaire.

Mnouchkine exacerbe donc ce phénomène d'illusion filmique afin de réduire l'écart entre la scène et l'écran lorsqu'elle remanie les décors du Dernier Caravansérail pour la création du film. Les décors pour le texte spectaculaire sont simplifiés car très nombreux, puisque le récit du spectacle suit un vaste mouvement spatio-temporel. L'histoire s'étale sur deux ans, de 2000 à 2002, et l'étendue géographique se situe entre des pays allant de l'Angleterre et la France jusqu'à la Nouvelle Zélande, en passant par l'Afghanistan, l'Iran, la Russie, la Bosnie-Herzégovine. Ainsi, pour les besoins matériels du spectacle, chaque décor constitue en lui-même une entité minimale qui doit être rapidement transportable par des machinistes et hors des murs de la Cartoucherie pour les tournées. L'essence de chaque décor est contenue sur des plateaux mobiles qui facilitent leurs entrées et sorties de la scène. L'univers spatio-temporel de ces plateaux mobiles est alors évoqué par un choix de traits distinctifs qui renvoient à un lieu précis et à un moment donné du récit - images documentaires défilant sur un téléviseur, costumes, langues parlées, etc. - ainsi que des signes de nature synecdotique une branche pour un arbre, une fenêtre sur un mur pour une demeure -, et encore des signes métaphoriques comme les effets sonores qui figurent une pluie battante qui n'atteint jamais les actants ou encore des véhicules aussi audibles qu'invisibles. Chaque plateau représente un concentré de natura- 


\section{Revista Brasileira de Estudos da Presenca Brazilitan Journal on Presence Studies}

lisme dont l'objectif est purement sémantique. L'imagination du public complète cet espace référentiel, contrastant avec le reste de la scène qui demeure, en comparaison, vide (Figure 9).

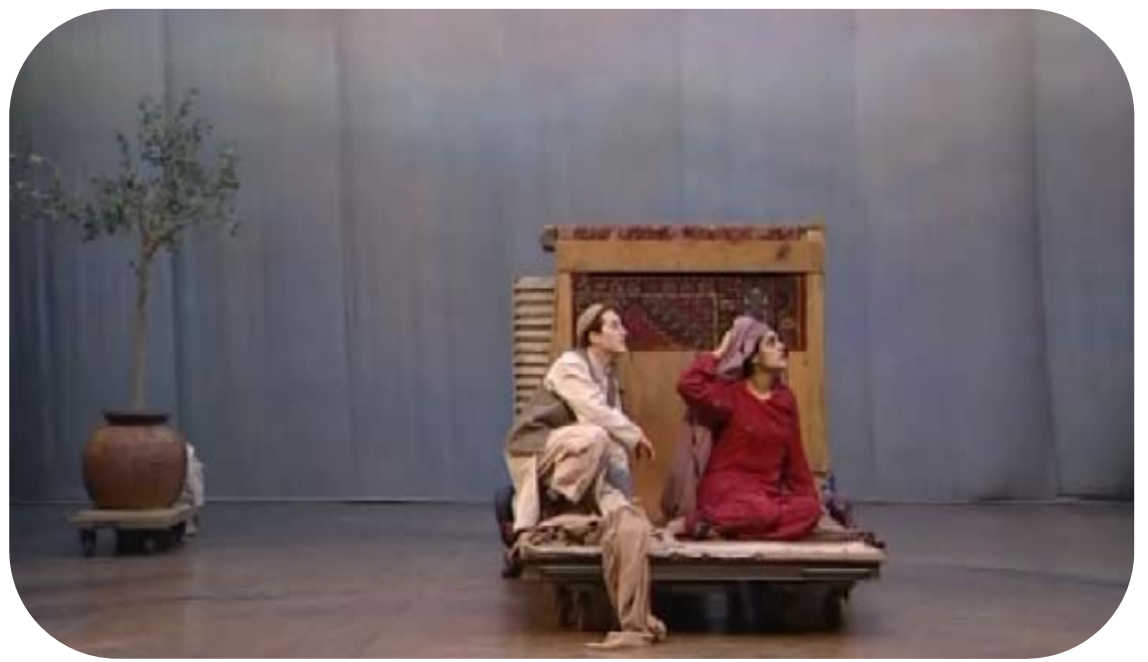

Figure 9 - Capture DVD. Complément de programme: Et au théâtre, c'était comment? Le Dernier Caravansérail, Ariane Mnouchkine (2006).

Or, la taille relativement réduite de ces décors aurait forcé l'utilisation systématique du gros plan lors de l'enregistrement sur support filmique. Mnouchkine donc prend la décision de filmer son spectacle en réinventant des décors mieux appropriés pour la rhétorique filmique - décision qui ne peut techniquement pas inclure un public in situ. Presque tous les décors subissent des transformations considérables pour la mise en film du spectacle: scènes de pluie torrentielle tangibles cette fois, neige artificielle qui recouvre la scène dans son entier, camions et bateaux grandeur nature, intérieurs et extérieurs extrêmement variés reflétant de manière minutieuse chaque pays et chaque culture. Le système d'effets spéciaux sonores et visuels est si subtil qu'il n'aurait pas été matériellement permis sur le plateau durant une présentation en public alors que le médium filmique permet une ubiquité qui n'est pas aussi transparente au théâtre du fait des intervalles occasionnés par les changements de décor. Sur l'écran, l'espace est à chaque fois comblé, les décors remplissent la totalité de l'objectif et produisent une forte impression de réalité.

Les scènes du film sont toutes tournées en intérieur à la Cartoucherie. Pourtant, les décors sont si réalistes, l'éclairage et les effets sonores si élaborés, que parfois l'illusion désoriente. Par exemple, il n'y a rien dans la première prise de vue de la scène initiale, avant que le plan ne s'élargisse sur les 


\section{Revista Brasileira

flots de soie, qui signale que la scène ne se joue pas en extérieur. Le court passage situé en Afrique recrée également à la perfection une scène de rue aussi animée que polluée de fumée et de bruits; et les scènes de nuits filmées en contre-plongée dévoilent des voûtes étoilées on ne peut plus réalistes (Figure 10).

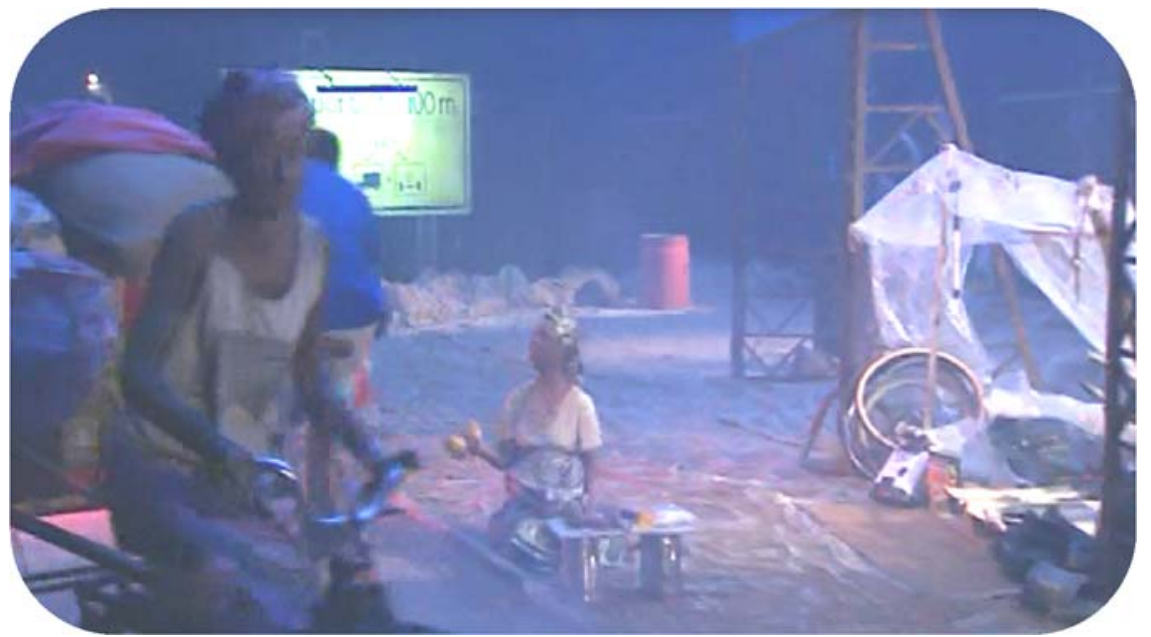

Figure 10 - Capture film, Le Dernier Caravansérail, Ariane Mnouchkine (2006).

Enfin, l'une des dernières scènes représente une femme dans une cabine téléphonique au bord de la Tamise. Sur le théâtre la femme semblait isolée, séparée du monde par l'immensité vide du plateau qui l'entourait seuls les effets sonores permettaient peut-être de combler ce vide et de se représenter mentalement les bords du fleuve londonien (Figure 11).

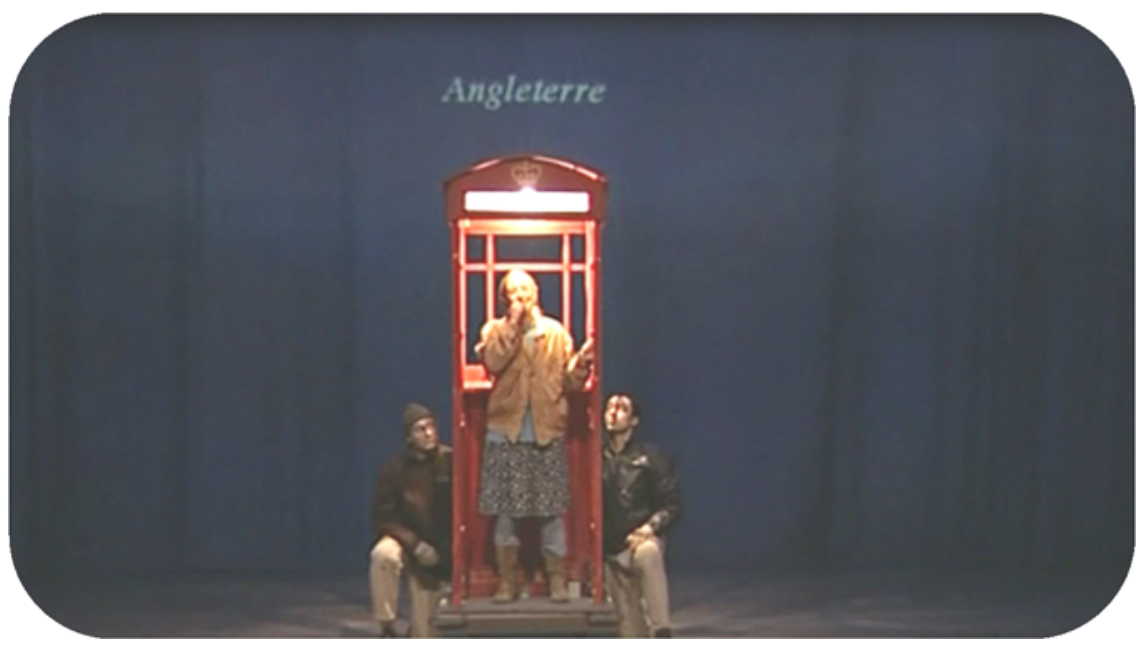

Figure 11 - Capture DVD. Complément de programme: Et au théâtre, c'était comment? Le Dernier Caravansérail, Ariane Mnouchkine (2006).

A l'écran, la situation est identique, la même femme converse dans la même cabine. Pourtant, notre œil est absolument dupé par le reflet des projecteurs - hors-champ - sur le tissu satiné du fleuve, la perspective créée par 


\section{Revista Brasileira de Estudos da Presenca Brazilien Journal on Presence Studies}

des bateaux miniatures dont les lumières scintillent au loin, la brise qui agite les cheveux du personnage, la subtilité sonore de la scène, qui font oublier que celle-ci prend place sur un théâtre (Figure 12).

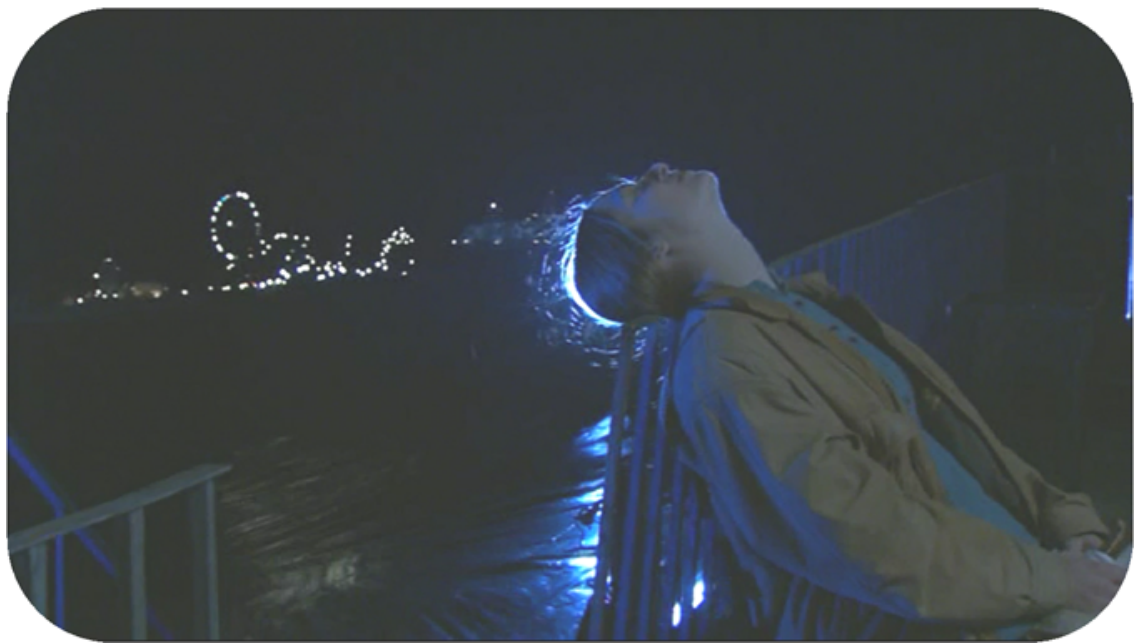

Figure 12 - Capture film, Le Dernier Caravansérail, Ariane Mnouchkine (2006).

L'illusion de réalité est accomplie par la finesse des effets spéciaux qui dupent les sens: cette femme semble effectivement se trouver au bord de la Tamise, comme elle l'a indiqué précédemment à son interlocuteur au téléphone. Cette scène bénéficie indéniablement du dispositif filmique capable de mettre en scène/ensemble les éléments de l'illusion dans l'objectif d'un désillusionnement sensoriel. Cette scène est un véritable coup de théâtre dont le plaisir esthétique s'amplifie de la réalisation que l'illusion, loin d'être étouffée par les possibilités filmiques, est non seulement revendiquée - à l'instar d'une hypotypose, on voit le fleuve grâce au son -, elle est surtout portée à son paroxysme: les sens ont dupé l'esprit.

Il est évident que la présence intrinsèque au texte spectaculaire - et les phénomènes qui s'y rapportent, aura, immédiateté, coprésence, sémiose et autopoïèse - est modifiée lors de son enregistrement filmique. Cependant, cette notion sur laquelle est fondée l'irréductible différence entre spectacle scénique et film-théâtre peut être nuancée, car les divers procédés mis en œuvre pour solliciter l'attention des publics de l'écran présentifient simultanément ceux qui leur sont absents, rendent présent à leur conscience le hic et nunc de ce qui leur est présenté.

Dans le cas du Bourgeois gentilhomme, les procédés d'amplification et de simulation ont permis d'accomplir des effets de présence par le biais d'un système de relais compétent, de signes spécifiquement filmiques. Fraudreau 
a pris en compte les moyens sémiotiques du théâtre (proxémie, kinésie et scénographie) et les a repris à son compte en privilégiant certains procédés de cinématographie et de mise en scène (montage, échelle de plan, cadrage) afin de mobiliser l'attention du public de l'écran et de recréer pour lui un effet de surprise similaire à celui vécu d'abord par le public in situ. Cet effet est vécu au présent du visionnement, bien que spatio-temporellement décalé par rapport au moment de sa présentation effective.

Quant aux moyens de focalisation conçus pour Phèdre ils ont permis de matérialiser la coprésence scène/salle lorsque les comédiens investissaient périodiquement l'espace conventionnellement dévolu à la salle - procédé dont se sert également Lagarce pour son interprétation de La Cantatrice chauve (2007). D'une part, ces interactions insistent sur la corporéité de l'acteur et renforcent la matérialité spatio-temporelle de l'événement spectaculaire ; d'autre part, l'empreinte physique de la relation entre la scène et la salle agit comme relais psychologique, incluant alors l'instance spectatrice filmique au sein de l'autopoï̀se théâtrale. Cette dernière n'est ainsi plus exclusive du texte scénique, mais est transposée sur le terrain du film-théâtre: bien que la spectatrice de l'écran ne soit pas dans la salle, elle est en mesure de s'y projeter.

Les films-théâtre de Mnouchkine cherchent également à privilégier la perspective de leurs spectateurs, bien que les deux exemples que j'ai choisis le montrent de manière différente: immersion totale dans le public de la salle pour 1789, et remaniement intégral du dispositif scénique pour Le Dernier Caravansérail. Le choix de modifier la scénographie de ce dernier, et de réinventer des décors mieux appropriés pour la rhétorique filmique, ne procède pas uniquement d'une nécessité fonctionnelle, mais résulte surtout d'une exigence à privilégier la perspective spectatorielle filmique. A l'instar de la notion de subterfuge avancée par Bourassa comme l'un des éléments clés des effets de présence ${ }^{14}$, Mnouchkine met à profit le caractère ontologique de l'image filmique afin de brouiller les limites entre voir et savoir: les yeux ne distinguent plus ce qui appartient au monde réel de ce qui est l'effet d'une illusion. Le spectateur sait que les images du monde qu'il voit sur l'écran sont le produit d'un artifice, d'une composition habile réalisée sur la scène d'un théâtre et pourtant, s'il en croit ses sens, ce qu'il voit semble bien réfléchir le monde réel. Cette scène illustre précisément la définition de l'effet de présence théorisé par Féral, à savoir que c'est sur cette dissociation 
que se fonde l'effet de présence. A l'instar de l'effet de réel conceptualisé par Roland Barthes ${ }^{15}$, les signes que sont les décors, les sons et les lumières agissent comme autant de subterfuges pour créer une illusion de réalité. Ceuxci, en réduisant l'écart entre illusion et réalité, non seulement facilitent la suspension volontaire de l'incrédulité, mais aussi tendent à rapprocher par voie de substitution le présent de la représentation de celui du visionnement de la représentation médiatisée.

J'aimerais conclure sur un plaidoyer en faveur du film-théâtre. Ainsi que cet article le démontre, si la notion de présence n'est pas impensable à l'écran, elle continue néanmoins de soutenir les discours préjudiciables au film-théâtre et ce, pour au moins deux raisons.

D'abord, ces discours reposent sur une comparaison déloyale. L'évaluation des spécificités génériques du texte scénique et du film-théâtre s'effectue souvent selon une liste de topoi récursifs creusant un fossé esthétique irréductible entre ces deux modes de présentation. Cependant, ces divergences sont évidentes et uniquement perçues comme négatives dans le contexte d'une comparaison qui n'a pas lieu d'être puisque texte scénique et film-théâtre constituent deux pratiques dont les spécificités seront à jamais distinctes - pour le plaisir de leurs adeptes respectifs, qui parfois même se confondent. Pourquoi comparer deux moyens d'expression dont les spécificités sont irréductibles? Pourquoi comparer des genres dont les traits n'existent que pour les distinguer les uns des autres? À moins de mettre en lumière les distinctions afin de mieux les apprécier, ce que Susan Stanford Friedman propose dans son article, Why not compare en énonçant une évidence: "Décrire une chose avec les termes d'une autre présuppose la connaissance de la première avec laquelle est comparée la seconde. L'élément connu opère comme moyen de comparaison avec l'élément inconnu, imposant une relation d'inégalité" (Friedman, 2011, p. 753-754) ${ }^{16}$. Le processus de comparaison n'est donc jamais neutre, et sous-tend souvent une volonté d'évaluation et de jugement au détriment du comparé ${ }^{17}$. Elle note pourtant que le refus de la comparaison peut amener à la perte de l'identification de nouvelles qualités que partagent deux objets comparés: “[...] mis côte à côte, chacun dans leur propre contexte, mais envisagés dans leur dis/similarité, les textes collationnés mettent au jour de nouvelles perspectives sur chacun, ainsi que des concepts inédits" (Friedman, 2011, p. 758-759) ${ }^{18}$. L'objectif de Friedman consiste donc à faire émerger les pouvoirs bénéfiques de 
l'activité comparative. Ainsi considérée, l'activité de comparer est créatrice de relativité, de nouveaux concepts, et surtout, elle abolit la hiérarchie. En effet, à quoi sert-il de comparer dans le seul objectif de renforcer les différences? La comparaison doit au contraire encourager la construction de nouvelles opinions, de manières inédites de penser. Dans ce contexte, l'activité comparative se dynamise, elle dépasse la mise au jour de distinctions basiques afin d'établir un dialogue entre médias hétérogènes. L'épreuve comparative se meut alors en une rencontre de réciprocités et non plus en un concours d'antagonismes. Par conséquent, comparer le texte dramatique et/ou le texte scénique avec le film-théâtre doit non seulement s'accompagner de l'acceptation de leurs disparités, mais surtout inciter à un enrichissement réciproque. Comme cet essai le confirme, certains praticiens du théâtre comme Mnouchkine exploitent ces distinctions afin de concevoir de nouveaux modes d'écriture.

Ensuite, ces discours ne prennent pas en compte un principe fondamental qui consiste à accepter les contraintes (re)présentatives inhérentes à un art qui en (re)présente un autre, à savoir qu'un texte spectaculaire soit (re)présenté à l'intérieur d'un régime (re)présentatif hétérogène avec toutes les modifications engendrées par les contraintes de ce passage d'un art à un autre. Dans Le Destin des images, Jacques Rancière questionne la notion d'irreprésentabilité de l'image à l'aide ce chiasme: “[...] il s'en déduit que la rupture avec la représentation en art n'est pas l'émancipation par rapport à la ressemblance mais bien l'émancipation de la ressemblance par rapport à [la représentation]". Il suggère ainsi "[...] qu'il n'y a pas d'irreprésentable comme propriété de l'événement. Il y a des choix" (Rancière, 2003, p. 136; p. 145). L'art du film-théâtre consiste à obéir aux règles du régime de présentation qui l'accueille, ce qui lui interdit par conséquent toute équation littérale avec une quelconque interprétation scénique puisque les spécificités de ce dernier sont inévitablement déplacées, formulées et configurées au sein d'un espace de présentation fondamentalement filmique. Comparer les phénomènes de présence à l'œuvre sur scène et sur l'écran ne fait alors sens que si l'on accepte que la présence inhérente au texte spectaculaire ne soit pas effectivement et littéralement traduisible par le film-théâtre, condition sine qua non pour celui-ci de revendiquer une expression qui lui est propre. Si la présence scénique dans ses manifestations multiples est a priori irréductible au médium filmique, et que l'immédiateté essentielle du texte specta- 
culaire n'est pas littéralement exprimable par le film-théâtre, celles-ci ne sont pas inconcevables car l'énonciation filmique permet de communiquer un degré de présence ${ }^{19}$, d'exprimer dûment le plaisir qui s'attache aux phénomènes de présence.

\section{Notes}

1 Je propose l'expression film-théâtre afin de mettre en lumière un critère de distinction commun à tous les films réalisés à partir de versions scéniques préexistantes. Dans son acception, le film-théâtre inclut toutes les formes possibles (avec/sans public, décors et distribution remaniés, etc.), du moment qu'il privilégie sa propre énonciation et le point de vue de son public. Voir: Siméon (2013).

2 Théâtre Trianon à Paris.

3 Le National Theater Live transmet, depuis 2009, les captations des productions du National Theater de Londres.

4 Pour plus de renseignements concernant les relations entre les publics de la salle et de l'écran, voir l'article d'Anna Andes (2017), The Instability of Suspending Audience Disbelief: Filming Productions at The Globe and The National Théâtre. Andes examine les différents choix que font les réalisateurs du NT Live et du Globe on Screen quant à la question de laisser paraître ou non le public à l'écran.

5 Nicolas Bataille est le premier metteur en scène du texte d'Ionesco.

6 Ces chaises n'ont pas de fonction pragmatique puisque personne ne s'y assoit, mais on une fonction dramaturgique: selon Chéreau, elles figurent le funeste du texte dramatique parce qu'elles sont laissées dans l'obscurité et renvoient ainsi métaphoriquement à la fatalité et à la mort.

7 Selon les catégories d'Edward T. Hall, l'espace interpersonnel est défini comme intime quand moins de 50 centimètres séparent deux individus. Voir: Pavis (1996).

8 Disponible sur: <http://www.theatre-du-soleil.fr/thsol/images/espaces-etscenographies/1789-1970-1124?lang=fr $>$.

9 Voir: André Bazin (1981).

10 Court métrage des Frères Lumière (1895).

${ }^{11}$ Avec l'aimable autorisation de l'Institut Lumière. 
${ }^{12}$ Avec l'aimable autorisation de l'Institut Lumière.

13 Alberti, Batista. De Pictura apud Jean-Claude Vuillemin (2010, p. 123).

14 “Si on parle d'effet', c'est que se glisse la notion d'un subterfuge qui trompe la perception" (Bourassa, 2013, p. 137).

15 Voir: Roland Barthes (1968).

16 Dans l'original en anglais: "In describing one thing in terms of another, comparison assumes knowledge of the one to which the other is compared. The known then operates as a measure of the unknown, standing in an unequal relation to it".

17 Friedman ajoute toutefois que l'acte de comparer est une attitude automatique qui permet de faire sens du monde, de rendre compréhensible ce qui ne l'est pas forcément.

18 Dans l'original en anglais: “[...] put side by side, each in its own distinctive context, but read together for their in/commensurability, texts in collage produce new insights about each, as well as new theoretical frameworks".

19 Josette Féral affirme que l'effet de présence est indépendant du type de dispositif. Voir: Josette Féral et Edwige Perrot (2012).

\section{Références}

1789. Réalisation: Ariane Mnouchkine. Paris: Le Théâtre du Soleil, 1973.

ANDES, Anna. The Instability of Suspending Audience Disbelief: Filming Productions at The Globe and The National Theater. In: SIMÉON, Sandrine; ALCAYAGA, Agathe Torti. Coup de Théâtre. Paris: Radac, 2017. P. 83-101.

BARTHES, Roland. L'Effet de Réel. Communications, Paris, v. 11, p. 84-89, 1968.

BAZIN, André. Théâtre et cinéma. Esprit, Paris, v. 19, p. 232-253, Août 1951.

BAZIN, André. Qu'est-ce que le cinéma? Paris: Editions du Cerf, 1981.

BENJAMIN, Walter. L'Guvre d'Art à l'Époque de sa Reproductibilité Technique. Paris: Gallimard, 2000.

BOUCHEZ, Pascal. Filmer l'Éphémère: récrire le théâtre (et Mesguich) en images et sons. Villeneuve d'Ascq: Septentrion, 2007.

BOURASSA, René. De la présence aux effets de présence: entre l'apparaître et l'apparence. In: FÉRAL, Josette; PERROT, Edwige. Le Réel à l'Ėpreuve des 
Technologies. Les Arts de la scène et les arts médiatiques. Rennes: Presses universitaires de Rennes, 2013. P. 129-148.

DELEUZE, Gilles. Cinéma 1: l'Image mouvement. Paris: Minuit, 1983.

FARCY, Gérard-Denis; PRÉDAL, René. Brûler les Planches, Crever l'Ècran. La présence de l'acteur. Montpellier: L'Entretemps, 2001.

FÉRAL, Josette; PERROT, Edwige. De la présence aux effets de présence. Ecarts et enjeux. In: FÉRAL, Josette. Pratiques Performatives, Body Remix. Rennes: Presses Universitaires de Rennes, 2012. P. 11-26.

FRANKENSTEIN. Réalisation: Tim Van Someren. London: National Theater Live, 2010.

FRIEDMAN, Susan Stanford. Why not compare? PMLA, New York, v. 123, n. 3, p. 753-760, May 2011.

GENETTE, Gérard. Figures III. Paris: Seuil, 1972.

HAMLET. Réalisation: Ricardo Aronovitch. London: BBC, 2002.

HAMLET. Réalisation: Robin Lough. London: National Theater Live, 2010.

IONESCO, Eugène. Notes et Contre-notes. Paris: Gallimard, 1975.

L'ARRIVÉE D'UN TRAIN en gare de la Ciotat. Réalisation: Frères Lumière. Paris: Lumière, 1895.

LA CANTATRICE Chauve. Réalisation: Vincent Bataillon. France: Arte, 2007.

LE BOURGEOIS Gentilhomme. Réalisation: Martin Fraudreau. France: Arte France, 2004. 1 DVD.

LE DERNIER Caravansérail. Réalisation: Ariane Mnouchkine. France: Bel Air Media, 2006. 1 DVD.

MOLIĖRE. Le Bourgeois Gentilhomme. In: FORESTIER, Georges (Ed.). Euvres Complètes. Paris: Gallimard, 2010.

PAVIS, Patrice. Dictionnaire du Théâtre. Paris: Dunod, 1996.

PHÈDRE. Réalisation: Stéphane Metge. France: Arte, 2003.

RACINE. Phèdre. In: FORESTIER, Georges (Ed.). CEuvres Complètes. Paris: Gallimard, 2010.

RANCIÈRE, Jacques. Le Destin des Images. Paris: Editions La Fabrique, 2003. 
SIMÉON, Sandrine. Le film-théâtre, un troisième temps du théâtre? In: ALCAYAGA, Agathe Torti; KIEHL, Agathe; KIEHL, Christine. Théâtre, Levain du Cinéma; théâtre, destin du cinéma. Paris: Le Manuscrit, 2013. P. 169-183.

VUILLEMIN, Jean-Claude. Réflexions sur la réflexivité théâtrale. L’Annuaire Théâtral, Montréal, SQET, v. 45, p. 119-135, 2010.

Sandrine Siméon est enseignante au Franklin \& Marshall College en Pennsylvanie où elle enseigne le français et le cinéma. Sa recherche se concentre sur le filmage du théâtre. Ses dernières publications incluent un volume pour la revue du Radac, Coup de théâtre, des articles sur le filmage du théâtre dans Coup de Théâtre, European Drama and Performance Studies, et dans Théatre, levain du cinéma; théatre, destin du cinéma.

E-mail: ssimeonf@fandm.edu

Ce texte inédit, révisé par André Mubarack, est également publié en portugais dans ce numéro.

Reçu le 29 novembre 2016 Accepté le 6 juin 2017 